\title{
Value of Cardiopulmonary Exercise Testing in the Prognosis Assessment of Chronic Obstructive Pulmonary Disease Patients: A Retrospective, Multicentre Cohort Study
}

\author{
Ralf Ewert ${ }^{a} \quad$ Anne Obst $^{a}$ Andreas Mühle ${ }^{b}$ Michael Halank ${ }^{c}$ Jörg Winkler ${ }^{d}$ \\ Bernd Trümper $^{\mathrm{e}}$ Gerhard Hoheisel $^{\mathrm{d}}$ Andreas Hoheisel ${ }^{f}$ Mark Wiersbitzky ${ }^{g}$ \\ Alexander Heine $^{\mathrm{a}} \quad$ Alexander Maiwald $^{\mathrm{a}}$ Sven Gläser ${ }^{\mathrm{a}, \mathrm{h}}$ Beate Stubbe ${ }^{\mathrm{a}}$

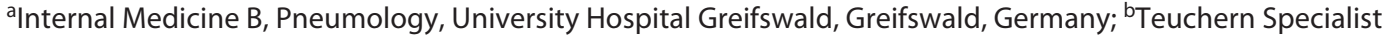 \\ Centre, Teuchern, Germany; 'Internal Medicine, Pneumology, University Hospital Dresden, Dresden, Germany; \\ ${ }^{\mathrm{d}}$ Medical Practice Leipzig, Leipzig, Germany; ${ }^{\mathrm{e}}$ Medical Practice Breathing \& Sleep Erfurt, Erfurt, Germany; \\ ${ }^{f}$ Clinic of Pneumology and Pulmonary Cell Research, University Hospital Basel, Basel, Switzerland; ${ }^{9}$ Medical Practice

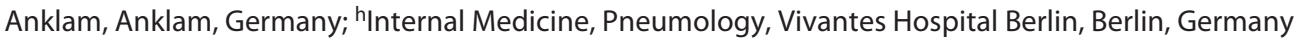

\section{Keywords}

Chronic obstructive pulmonary disease - Lung function . Cardiopulmonary exercise testing · Prognosis · All-cause mortality

\begin{abstract}
Introduction: Chronic obstructive pulmonary disease (COPD) is one of the most common chronic diseases associated with high mortality. Previous studies suggested a prognostic role for peak oxygen uptake $\left(\mathrm{VO}_{2}\right.$ peak) assessed during cardiopulmonary exercise testing (CPET) in patients with COPD. However, most of these studies had small sample sizes or short follow-up periods, and despite their relevance, CPET parameters are not included in the Global Initiative for Chronic Obstructive Lung Disease (GOLD) tool for assessment of severity. Objectives: We therefore aimed to assess the prog-
\end{abstract}

nostic value of CPET parameters in a large cohort of outpatients with COPD. Methods: In this retrospective, multicentre cohort study, medical records of patients with COPD who underwent CPET during 2004-2017 were reviewed and demographics, smoking habits, GOLD grade and category, exacerbation frequency, dyspnoea score, lung function measurements, and CPET parametersweredocumented.Relationships with survival were evaluated using Kaplan-Meier analysis, Cox regression, and receiver operating characteristic (ROC) curves. Results: Of a total of 347 patients, 312 patients were included. Five-year and 10-year survival probability was $75 \%$ and $57 \%$, respectively. $\mathrm{VO}_{2}$ peak significantly predicted survival (hazard ratio: 0.886 [95\% confidence interval: 0.830 ; 0.946]). The optimal $\mathrm{VO}_{2}$ peak threshold for discrimination of 5-year survival was $14.6 \mathrm{~mL} / \mathrm{kg} / \mathrm{min}$ (area under ROC curve: 0.713 ). Five-year survival in patients with $\mathrm{VO}_{2}$ peak $<14.6 \mathrm{~mL} /$ $\mathrm{kg} / \mathrm{min}$ versus $\geq 14.6 \mathrm{~mL} / \mathrm{kg} / \mathrm{min}$ was $60 \%$ versus $86 \%$ in karger@karger.com www.karger.com/res

Karger $\stackrel{\text { ' }}{5}$

GOPEN ACCESS
(C) 2021 The Author(s)

Published by S. Karger AG, Basel

This article is licensed under the Creative Commons Attribution 4.0 International License (CC BY) (http://www.karger.com/Services/ OpenAccessLicense). Usage, derivative works and distribution are permitted provided that proper credit is given to the author and the original publisher.
Correspondence to:

Beate Stubbe, beate.stubbe@uni-greifswald.de 
GOLD categories $A / B$ and $64 \%$ versus $90 \%$ in GOLD categories C/D. Conclusions: We confirm that $\mathrm{VO}_{2}$ peak is a highly significant predictor of survival in COPD patients and recommend the incorporation of $\mathrm{VO}_{2}$ peak into the assessment of COPD severity.

(c) 2021 The Author(s).

Published by S. Karger AG, Basel

\section{Introduction}

Chronic obstructive pulmonary disease (COPD) is one of the most common chronic diseases worldwide, affecting 105 million men and 70 million women [1]. The severity of COPD is graded based on airflow limitation (measured as forced expiratory volume in $1 \mathrm{~s}\left[\mathrm{FEV}_{1}\right]$ ) [2], but prognosis in COPD is not determined by lung function limitations alone. Compared with the general population, patients with COPD have higher rates of comorbidity, especially cardiac disease, diabetes mellitus, lung cancer, and depression $[3,4]$. These comorbidities significantly influence mortality $[5,6]$ as well as cardiorespiratory fitness and physical activity [7]. Physical activity in turn significantly influences the course of COPD (decline of lung function) [8] and survival in patients with COPD [9]. Cardiopulmonary exercise testing (CPET) has prov- en to be an important non-invasive tool for assessing patients with various pulmonary diseases, such as COPD, interstitial lung disease, or cystic fibrosis [10-16].

In patients with COPD, multidimensional indices have been established for the evaluation of prognosis [17]. Some of these indices include parameters of cardiorespiratory fitness, such as the 6-min walking distance or peak oxygen uptake ( $\mathrm{VO}_{2}$ peak) assessed during CPET [18]. The prognostic value of CPET parameters in patients with COPD has been reported in several studies [19-24], but most of these studies had small sample sizes and only a few had an observation time exceeding 5 years [21-23], and despite their relevance only few CPET parameters were currently included. Further, CPET variables were not included in key algorithms for assessment of COPD severity $[2,25]$. The aim of this multicentre, retrospective, observational study was to assess the longterm prognostic value of CPET-derived parameters in a large cohort of outpatients with COPD.

\section{Patients/Methods}

Patients

Patients with COPD of all grades who had undergone CPET during the period January 2004-December 2017 were retrospec-

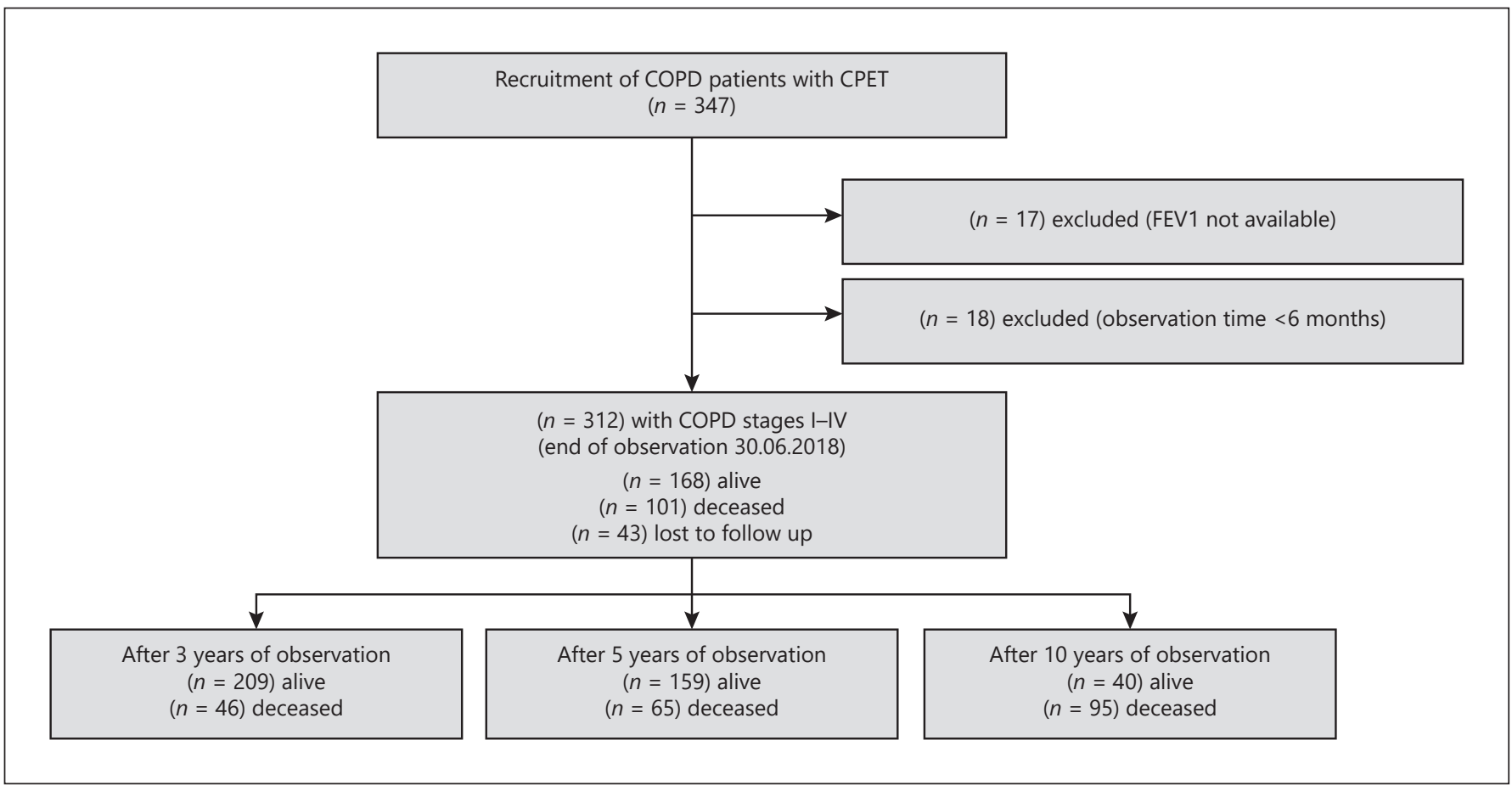

Fig. 1. Patient flow. COPD, chronic obstructive pulmonary disease; CPET, cardiopulmonary exercise testing; $\mathrm{FEV}_{1}$, forced expiratory volume in $1 \mathrm{~s}$. 
tively included from different pulmonary specialist practices in Germany. Exclusion criteria were coincident other airway or pulmonary diseases, chronic hypercapnic respiratory insufficiency with arterial partial pressure of carbon dioxide $>50 \mathrm{~mm} \mathrm{Hg}$ at rest, acute COPD exacerbations, necessary change of long-term medication in the 6 weeks prior to inclusion, unstable coronary disease necessitating intervention in the previous 6 months, impaired left ventricular pump function (ejection fraction $<50 \%$ ) measured by echocardiogram, clinical signs of right heart overload, and uncontrolled comorbidities. Enrolled patients were excluded from the analysis if their observation period was $<6$ months or if they did not have a post-bronchodilatation $\mathrm{FEV}_{1}$ value recorded.

\section{Data Collection}

The following information was collected from the medical records of participating patients: gender, age, body mass index (BMI), smoking history, number of exacerbations in the year preceding lung function testing and CPET, lung function parameters, and CPET data. Lung function parameters were calculated relative to reference values as described previously [26-28]. CPET parameters were obtained using a modified Jones protocol ( 3 min rest, 1 min unloaded cycling, stepwise increase in workload of $16 \mathrm{Watt} /$ min, starting with unloaded cycling plus the ergometer-related permanent load, 5 min recovery) on a braked cycle ergometer, as described previously [29]. All technical staff in the participating centres underwent specific CPET training, and the evaluation of CPET data was performed centrally by few experienced physicians. If a patient had undergone CPET multiple times during the study period, only the first CPET was included for analysis. The severity grading of COPD followed the Global Initiative for Chronic Obstructive Lung Disease (GOLD) grades I-IV and GOLD categories A-D [2] as well as the modified BMI, airflow obstruction, dyspnoea, and exercise capacity (mBODE) index [18], the age, dyspnoea, and airflow obstruction index [30], and the dyspnoea, airflow obstruction, current smoking status, and exacerbations index [31]. The degree of dyspnoea was assessed by the modified MRC scale.

The participating patients were followed until June 30, 2018. Survival was determined by contacting the patient or their local physician. Patients who could not be contacted were classed as lost to follow-up.

\section{Statistical Analyses}

Statistical analyses were performed with SAS 9.4 (SAS Institute Inc., Cary, NC, USA); $p<0.05$ was considered statistically significant. No sample size calculation was performed; all patients with available data who underwent CPET at participating centres were included. Differences between groups were analysed by the Wilcoxon test. The analysis of survival data followed the Kaplan-Meier method. To identify predictors of survival, models were determined using Cox regression with elimination of variables by selection procedures using a cut-off $p$ value of 0.05 . For selected parameters, optimal cut-offs for prediction of survival were identified using receiver operating characteristic (ROC) curves and the Youden index.

\section{Analysis of Literature}

We searched PubMed for observational studies of the prognostic value of CPET parameters in COPD published up to Dec 10, 2020, with the following terms in the title/abstract: (cardiopulmo-
Table 1. Patient characteristics

\begin{tabular}{|c|c|}
\hline & $\begin{array}{l}\text { Patients with COPD } \\
(n=312)\end{array}$ \\
\hline Female & $81(26.0)$ \\
\hline Age at time of CPET, years & $65 \pm 9$ \\
\hline $\mathrm{BMl}, \mathrm{kg} / \mathrm{m}^{2}$ & $26.9 \pm 5.2$ \\
\hline Time since COPD diagnosis, years $(n=171)$ & $8 \pm 7$ \\
\hline \multicolumn{2}{|l|}{ Smoking status $(n=299)$} \\
\hline Never-smoker & $36(12.0)$ \\
\hline Smoker & $112(37.5)$ \\
\hline Ex-smoker & $151(50.5)$ \\
\hline \multicolumn{2}{|l|}{ Pack years available } \\
\hline Smoker $(n=84)$ & $38 \pm 14$ \\
\hline Ex-smoker $(n=150)$ & $38 \pm 21$ \\
\hline \multicolumn{2}{|l|}{ COPD grade (GOLD) } \\
\hline 1 & $9(2.9)$ \\
\hline II & $86(27.6)$ \\
\hline III & $170(54.5)$ \\
\hline IV & $47(15.1)$ \\
\hline \multicolumn{2}{|l|}{ COPD category $(n=242)$} \\
\hline A & $15(6.2)$ \\
\hline $\mathrm{B}$ & $49(20.2)$ \\
\hline $\mathrm{C}$ & $16(6.6)$ \\
\hline $\mathrm{D}$ & $162(66.9)$ \\
\hline \multicolumn{2}{|l|}{ Dyspnoea scale (mMRC; $n=275)$} \\
\hline 1 & $33(12.0)$ \\
\hline 2 & $98(35.6)$ \\
\hline 3 & $128(46.5)$ \\
\hline 4 & $16(5.8)$ \\
\hline \multicolumn{2}{|l|}{ Exacerbations in previous year $(n=223), n$} \\
\hline $0-1$ & $153(68.6)$ \\
\hline$>1$ & $70(31.4)$ \\
\hline mBODE index $(n=275)$ & $4.79(2.25)$ \\
\hline 0 & $5(1.8)$ \\
\hline 1 & $22(8.0)$ \\
\hline 2 & $24(8.7)$ \\
\hline 3 & $28(10.2)$ \\
\hline 4 & $39(14.2)$ \\
\hline 5 & $42(15.3)$ \\
\hline 6 & $48(17.5)$ \\
\hline 7 & $41(14.9)$ \\
\hline 8 & $14(5.1)$ \\
\hline 9 & $8(2.9)$ \\
\hline 10 & $4(1.5)$ \\
\hline ADO index $(n=275)$ & $4.66(1.51)$ \\
\hline 0 & $1(0.4)$ \\
\hline 1 & $9(3.3)$ \\
\hline 2 & $14(5.1)$ \\
\hline 3 & 35 (12.7) \\
\hline 4 & 52 (18.9) \\
\hline 5 & $78(28.4)$ \\
\hline 6 & $61(22.2)$ \\
\hline 7 & $23(8.4)$ \\
\hline 8 & $2(0.7)$ \\
\hline DOSE index $(n=196)$ & $3.09(1.47)$ \\
\hline 0 & $6(3.1)$ \\
\hline 1 & $29(14.8)$ \\
\hline 2 & $27(13.8)$ \\
\hline 3 & $55(28.1)$ \\
\hline 4 & $47(24.0)$ \\
\hline 5 & $24(12.2)$ \\
\hline 6 & $6(3.1)$ \\
\hline 7 & $2(1.0)$ \\
\hline
\end{tabular}

Data are presented as mean \pm standard deviation or $n$ (\%). BMI, body mass index; COPD, chronic obstructive pulmonary disease; CPET, cardiopulmonary exercise testing; GOLD, global initiative for chronic obstructive lung disease; mMRC, modified medical research council; $\mathrm{mBODE}$, modified $\mathrm{BMl}$, airflow obstruction, dyspnoea, and exercise capacity; ADO, age, dyspnoea, and airflow obstruction; DOSE, dyspnoea, airflow obstruction, current smoking status, and exacerbations. 
nary exercise test OR cardiopulmonary exercise tests OR cardiopulmonary exercise testing OR CPET OR pulmonary exercise test OR pulmonary exercise tests OR pulmonary exercise testing OR oxygen uptake $\mathrm{OR}_{2}$ uptake $\mathrm{OR}$ oxygen consumption $\mathrm{OR}_{2}$ consumption $\mathrm{OR} \mathrm{VO}_{2} \mathrm{OR} \mathrm{VO}_{2} \max$ ) AND (mortality OR survival OR prognosis OR prognostic OR death OR deaths) AND (chronic obstructive pulmonary disease OR COPD). Studies focusing on a specific comorbidity associated with COPD were excluded. The identified studies suggested a prognostic role for $\mathrm{VO}_{2}$ peak and other variables assessed during CPET in patients with COPD. However, CPET parameters have not yet been included in the GOLD tool for assessment of COPD severity or prognosis.

\section{Results}

\section{Patients}

In total, 347 patients with COPD were enrolled (Fig. 1). We excluded 18/347 patients because their observation period was $<6$ months, and $17 / 347$ patients because the post-bronchodilatation $\mathrm{FEV}_{1}$ value was missing. The remaining 312 patients (mean \pm standard deviation [SD] observation time: $67 \pm 44$ months [range: $6-170$ months]) were included in the analysis. By the end of the study (June 30, 2018), 43/347 patients could not be contacted and were therefore lost to follow-up.

Patient characteristics are shown in Table 1. The mean age of the patients at the time of inclusion into the study was $65 \pm 9$ years, and the majority were male (74\%). Most patients (54\%) had COPD in GOLD grade III, indicating severe airflow limitation.

Lung function values and CPET results are summarized in Table 2. The mean $\mathrm{FEV}_{1}$ value indicated severe airflow limitation. Pulmonary exercise limitation (ratio of minute ventilation [VÉ] to maximum voluntary ventilation $>80 \%$ ) was detected in $48 \%$ of the patients. Impaired ventilatory efficiency was detected in $45 \%$ of the patients when defined as a VÉ/carbon dioxide output $\left(\mathrm{VCO}_{2}\right)$ slope of $>34$, and in $58 \%$ of the patients when defined as a $\mathrm{VE} / \mathrm{VCO}_{2}$ nadir $>34$.

\section{Survival}

After 3, 5, and 10 years, the survival probability was $83 \%, 75 \%$, and $57 \%$, respectively. Patient characteristics, lung function parameters, and CPET results were compared between those who had died and those who were still alive at each of the 3 follow-up time points (Tables 3 $-5)$. In all 3 comparisons, the deceased patients were significantly older at study entry and had significantly lower global diffusion capacity, cardiopulmonary capacity (maximum power, $\mathrm{VO}_{2}$ peak, and $\mathrm{VO}_{2} @ \mathrm{AT}$ ), end-tidal pressure of carbon dioxide @AT (petCO $\mathrm{A}_{2} \mathrm{AT}$ ), ventila-
Table 2. Pulmonary function and CPET parameters

Patients

with COPD

$(n=312)$

Pulmonary function

$\mathrm{FEV}_{1} / \mathrm{FVC}_{1} \%(n=308)$

$\mathrm{FEV}_{1}, \%$ predicted

$55.2 \pm 14.0$

VC, $\%$ predicted $(n=308)$

$\mathrm{RV}, \%$ predicted $(n=264)$

RV/TLC, \% $(n=262)$

$45.6 \pm 16.4$

$68.4 \pm 17.2$

TLC, \% predicted $(n=264)$

$206.8 \pm 66.0$

$162.7 \pm 29.2$

DLCO, \% predicted $(n=149)$

$117.9 \pm 23.0$

KCO, \% predicted $(n=152)$

$39.4 \pm 15.9$

CPET

Maximum power, \% predicted* $(n=311) \quad 53.0 \pm 16.9$

$\mathrm{VO}_{2}$ peak, \% predicted*

$58.3 \pm 17.0$

$\mathrm{VO}_{2} @ \mathrm{AT}, \%$ predicted* $(n=265)$

VO, peak/HRpeak, \% predicted* $(n=309) \quad 72.0 \pm 18.9$

VÉpeak, \% predicted* $(n=310)$

VÉ/MVV, \% $(n=308)$

BFpeak, breaths/min $(n=310)$

VÉ/VCO $\mathrm{V}_{2}$ slope $(n=300)$

Proportion of patients with VÉ/ $/ \mathrm{VCO}_{2}$ slope $>34$

petCO $2 @ A T, m m H g(n=260)$

VÉ/VCO 2 nadir $(n=270)$

Proportion of patients with VÉ/VCO $2 @ A T>34$

$149(48.4)$

$32 \pm 7$

$35 \pm 10$

$135(45.0)$

$34.0 \pm 6.1$

$36.7 \pm 7.7$

$157(58.1)$

Data are presented as mean \pm standard deviation or $n$ (\%). BFpeak, peak breathing frequency; CPET, cardiopulmonary exercise testing; COPD, chronic obstructive pulmonary disease; DLCO, diffusion capacity of the lung for carbon monoxide; $F E V_{1}$. forced expiratory volume in $1 \mathrm{~s}$; FVC, forced vital capacity; KCO, Krogh factor (diffusion capacity of the lung for carbon monoxide per alveolar volume); PetCO $\mathrm{C}_{2} @ \mathrm{AT}$, end-tidal pressure of carbon dioxide at anaerobic threshold; RV, residual volume; TLC, total lung capacity; VC, vital capacity; VÉ/MVV, ratio of ventilation to maximum voluntary ventilation; VÉpeak, peak ventilation; VÉ/ $/ \mathrm{CO}_{2}$ nadir, ratio of ventilation to carbon dioxide output; VÉ/VCO $\mathrm{VO}_{2}$ slope, slope of the relation between ventilation and carbon dioxide output; $\mathrm{VO}_{2} @ \mathrm{AT}$, oxygen uptake at anaerobic threshold; $\mathrm{VO}_{2}$ peak, peak oxygen uptake; $\mathrm{VO}_{2}$ peak/HRpeak, ratio of peak oxygen uptake to peak heart rate. ${ }^{*}$ Reference values were calculated as described [29] (see online suppl. Table 1 for details).

tory efficiency $\left(\mathrm{VÉ} / \mathrm{VCO}_{2}\right.$ slope and $\mathrm{VÉ} / \mathrm{VCO}_{2}$ nadir), oxygen pulse $\left(\mathrm{VO}_{2}\right.$ peak/peak heart rate [HRpeak]), and maximum ventilation (VÉpeak) compared with the surviving patients. In the comparisons at 5 and 10 years (but not 3 years) of follow-up, those who had died had a lower $\mathrm{BMI}, \mathrm{FEV}_{1}$, and $\mathrm{FEV}_{1}$ /forced vital capacity ratio than those who were still alive. In addition, volume-adapted diffusion capacity was significantly lower in those who had died compared with those who were still alive at 5 years, and vital capacity was significantly lower and VÉ/ maximum voluntary ventilation was significantly higher in those who had died compared with those who were still alive at 10 years.

Patients with COPD in GOLD grade I had 100\% survival at 10 years (Fig. 2). Survival at 3, 5, and 10 years was $80 \%, 74 \%$, and $66 \%$, respectively, in patients with grade II COPD, $86 \%, 79 \%$, and $51 \%$, respectively, in patients with grade III COPD, and $74 \%, 56 \%$, and $40 \%$, respectively, in 
Table 3. Characteristics, pulmonary function, and CPET parameters of patients stratified by survival status at 3 years

\begin{tabular}{|c|c|c|c|c|c|}
\hline & \multicolumn{2}{|c|}{ Alive $(n=209)$} & \multicolumn{2}{|c|}{ Deceased $(n=46)$} & \multirow[t]{2}{*}{$p$ value } \\
\hline & $n$ & mean $\pm S D$ & $n$ & mean $\pm S D$ & \\
\hline Age at time of CPET, years & 209 & $64 \pm 10$ & 46 & $68 \pm 8$ & 0.018 \\
\hline $\mathrm{BMI}, \mathrm{kg} / \mathrm{m}^{2}$ & 209 & $27.4 \pm 4.9$ & 46 & $25.7 \pm 5.7$ & 0.054 \\
\hline Time since COPD diagnosis, years & 126 & $9 \pm 7$ & 25 & $11 \pm 8$ & 0.25 \\
\hline \multicolumn{6}{|l|}{ Pulmonary function } \\
\hline $\mathrm{FEV}_{1} / \mathrm{FVC}, \%$ & 205 & $56.5 \pm 13.4$ & 46 & $53.0 \pm 13.7$ & 0.16 \\
\hline $\mathrm{FEV}_{1}, \%$ predicted & 209 & $46.6 \pm 16.2$ & 46 & $42.0 \pm 14.0$ & 0.11 \\
\hline VC, \% predicted & 205 & $68.3 \pm 17.4$ & 46 & $65.6 \pm 16.3$ & 0.50 \\
\hline RV, \% predicted & 168 & $210.9 \pm 58.0$ & 41 & $195.6 \pm 79.0$ & 0.16 \\
\hline RV/TLC, \% & 167 & $166.0 \pm 27.1$ & 41 & $157.0 \pm 33.6$ & 0.14 \\
\hline TLC, \% predicted & 168 & $119.3 \pm 20.5$ & 41 & $111.6 \pm 27.6$ & 0.10 \\
\hline DLCO, \% predicted & 91 & $40.6 \pm 16.5$ & 23 & $32.6 \pm 12.1$ & 0.019 \\
\hline KCO, \% predicted & 93 & $54.8 \pm 20.9$ & 23 & $46.5 \pm 17.1$ & 0.082 \\
\hline \multicolumn{6}{|l|}{ CPET } \\
\hline Maximum power, \% predicted* & 208 & $55.2 \pm 17.0$ & 46 & $47.2 \pm 17.7$ & 0.021 \\
\hline $\mathrm{VO}_{2}$ peak, \% predicted* & 209 & $61.9 \pm 16.5$ & 46 & $50.3 \pm 16.5$ & $<0.0001$ \\
\hline VO $\mathrm{VOAT}_{2}$ \% predicted* & 177 & $75.4 \pm 17.6$ & 39 & $64.6 \pm 16.1$ & 0.0003 \\
\hline $\mathrm{VO}_{2}$ peak/HRpeak, \%predicted* & 207 & $75.8 \pm 18.4$ & 45 & $61.4 \pm 18.9$ & $<0.0001$ \\
\hline VÉpeak, \% predicted* & 208 & $65.9 \pm 17.4$ & 45 & $58.0 \pm 16.7$ & 0.011 \\
\hline VÉ/MVV, \% & 206 & $80.5 \pm 19.3$ & 45 & $78.7 \pm 19.3$ & 0.42 \\
\hline BFpeak, breaths/min & 208 & $31 \pm 6$ & 45 & $32 \pm 7$ & 0.62 \\
\hline VÉ/VCO 2 slope & 201 & $34 \pm 9$ & 43 & $37 \pm 10$ & 0.031 \\
\hline petCO $2 @ A T, m m ~ H g$ & 176 & $35.1 \pm 6.2$ & 35 & $32.2 \pm 5.8$ & 0.016 \\
\hline VÉ/VCO ${ }_{2}$ nadir & 184 & $35.1 \pm 6.8$ & 37 & $39.9 \pm 9.1$ & 0.0016 \\
\hline
\end{tabular}

BFpeak, peak breathing frequency; BMI, body mass index; COPD, chronic obstructive pulmonary disease; CPET, cardiopulmonary exercise testing; DLCO, diffusion capacity of the lung for carbon monoxide; FEV 1 , forced expiratory volume in $1 \mathrm{~s}$; FVC, forced vital capacity; KCO, Krogh factor (diffusion capacity of the lung for carbon monoxide per alveolar volume); PetCO $\mathrm{C}_{2} @ \mathrm{AT}$, end-tidal pressure of carbon dioxide at anaerobic threshold; RV, residual volume; SD, standard deviation; TLC, total lung capacity; VC, vital capacity; VÉ/MVV, ratio of ventilation to maximum voluntary ventilation; VÉpeak, peak ventilation; VÉ/VCO 2 nadir, ratio of ventilation to carbon dioxide output; VÉ/VCO ${ }_{2}$ slope, slope of the relation between ventilation and carbon dioxide output; $\mathrm{VO}_{2} @ \mathrm{AT}$, oxygen uptake at anaerobic threshold; $\mathrm{VO}_{2}$ peak, peak oxygen uptake; $\mathrm{VO}_{2}$ peak/ $\mathrm{HRpeak}$, ratio of peak oxygen uptake to peak heart rate. *Reference values were calculated as described [29] (see online suppl. Table 1 for details).

Table 4. Characteristics, pulmonary function, and CPET parameters of patients stratified by survival status at 5 years

\begin{tabular}{|c|c|c|c|c|c|}
\hline & \multicolumn{2}{|c|}{ Alive $(n=159)$} & \multicolumn{2}{|c|}{ Deceased $(n=65)$} & \multirow[t]{2}{*}{$p$ value } \\
\hline & $n$ & mean $\pm S D$ & $n$ & mean $\pm S D$ & \\
\hline Age at time of CPET, years & 159 & $64 \pm 10$ & 65 & $69 \pm 8$ & 0.0013 \\
\hline $\mathrm{BMI}, \mathrm{kg} / \mathrm{m}^{2}$ & 159 & $27.6 \pm 4.7$ & 65 & $25.8 \pm 5.2$ & 0.011 \\
\hline Time since COPD diagnosis, years & 104 & $9 \pm 7$ & 39 & $11 \pm 7$ & 0.27 \\
\hline \multicolumn{6}{|l|}{ Pulmonary function } \\
\hline $\mathrm{FEV}_{1} / \mathrm{FVC}_{1} \%$ & 156 & $57.0 \pm 13.0$ & 65 & $52.2 \pm 13.6$ & 0.019 \\
\hline $\mathrm{FEV}_{1}, \%$ predicted & 159 & $47.2 \pm 15.4$ & 65 & $41.5 \pm 14.2$ & 0.013 \\
\hline VC, $\%$ predicted & 156 & $68.6 \pm 17.5$ & 65 & $65.5 \pm 17.2$ & 0.25 \\
\hline $\mathrm{RV}, \%$ predicted & 124 & $209.4 \pm 57.5$ & 58 & $201.6 \pm 75.1$ & 0.52 \\
\hline $\mathrm{RV} / \mathrm{TLC}, \%$ & 123 & $165.8 \pm 26.7$ & 58 & $160.1 \pm 33.0$ & 0.53 \\
\hline TLC, \% predicted & 124 & $119.2 \pm 20.9$ & 58 & $113.6 \pm 26.2$ & 0.28 \\
\hline DLCO, \% predicted & 64 & $42.4 \pm 15.6$ & 32 & $31.9 \pm 13.3$ & 0.0010 \\
\hline $\mathrm{KCO}, \%$ predicted & 66 & $56.7 \pm 20.2$ & 32 & $44.8 \pm 17.7$ & 0.0063 \\
\hline \multicolumn{6}{|l|}{ CPET } \\
\hline Maximum power, \% predicted* & 158 & $57.3 \pm 16.4$ & 65 & $47.1 \pm 17.6$ & $<0.0001$ \\
\hline $\mathrm{VO}_{2}$ peak, \% predicted* & 159 & $63.6 \pm 15.8$ & 65 & $50.8 \pm 16.6$ & $<0.0001$ \\
\hline $\mathrm{VO}_{2} @ \mathrm{AT}, \%$ predicted* & 139 & $76.9 \pm 16.6$ & 54 & $64.3 \pm 17.4$ & $<0.0001$ \\
\hline $\mathrm{VO}_{2}$ peak/HRpeak, $\%$ predicted* & 157 & $76.7 \pm 17.6$ & 64 & $62.7 \pm 20.6$ & $<0.0001$ \\
\hline VÉpeak, \% predicted* & 158 & $66.8 \pm 16.8$ & 64 & $58.9 \pm 16.9$ & 0.0044 \\
\hline VÉ/MVV, \% & 157 & $81.0 \pm 19.5$ & 64 & $80.0 \pm 18.8$ & 0.96 \\
\hline BFpeak, breaths/min & 158 & $31 \pm 6$ & 64 & $32 \pm 6$ & 0.18 \\
\hline VÉ/VCO 2 slope & 154 & $34 \pm 10$ & 59 & $37 \pm 9$ & 0.0021 \\
\hline petCO $2 @ A T, m m ~ H g$ & 137 & $35.5 \pm 6.0$ & 49 & $32.3 \pm 5.8$ & 0.0007 \\
\hline VÉ/NCO ${ }_{2}$ nadir & 144 & $34.6 \pm 6.8$ & 52 & $39.6 \pm 8.3$ & $<0.0001$ \\
\hline
\end{tabular}

BFpeak, peak breathing frequency; BMI, body mass index; COPD, chronic obstructive pulmonary disease; CPET, cardiopulmonary exercise testing; DLCO, diffusion capacity of the lung for carbon monoxide; $\mathrm{FEV}_{1}$, forced expiratory volume in $1 \mathrm{~s}$; FVC, forced vital capacity; KCO, Krogh factor (diffusion capacity of the lung for carbon monoxide per alveolar volume); PetCO $\mathrm{C}_{2} @ \mathrm{AT}$, end-tidal pressure of carbon dioxide at anaerobic threshold; RV, residual volume; SD, standard deviation; TLC, total lung capacity; VC, vital capacity; VÉ/MVV, ratio of ventilation to maximum voluntary ventilation; VÉpeak, peak ventilation; VÉ/ $\mathrm{VCO}_{2}$ nadir, ratio of ventilation to carbon dioxide output; VÉ/VCO 2 slope, slope of the relation between ventilation and carbon dioxide output; $\mathrm{VO}_{2} @ \mathrm{AT}$, oxygen uptake at anaerobic threshold; $\mathrm{VO}_{2}$ peak, peak oxygen uptake; $\mathrm{VO}_{2}$ peak/ $\mathrm{HRpeak}$, ratio of peak oxygen uptake to peak heart rate. * Reference values were calculated as described [29] (see online suppl. Table 1 for details).

Cardiopulmonary Exercise Testing in COPD Patients
Respiration 2022;101:353-366

DOI: $10.1159 / 000519750$ 
Table 5. Characteristics, pulmonary function, and CPET parameters of patients stratified by survival status at 10 years

\begin{tabular}{|c|c|c|c|c|c|}
\hline & \multicolumn{2}{|c|}{ Alive $(n=40)$} & \multicolumn{2}{|c|}{ Deceased $(n=95)$} & \multirow[t]{2}{*}{$p$ value } \\
\hline & $n$ & mean $\pm S D$ & $n$ & mean $\pm S D$ & \\
\hline Age at time of CPET, years & 40 & $63 \pm 10$ & 95 & $68 \pm 8$ & 0.0058 \\
\hline $\mathrm{BMI}, \mathrm{kg} / \mathrm{m}^{2}$ & 40 & $28.6 \pm 4.4$ & 95 & $26.4 \pm 5.0$ & 0.022 \\
\hline Time since COPD diagnosis, years & 4 & $12 \pm 6$ & 59 & $10 \pm 7$ & 0.58 \\
\hline \multicolumn{6}{|l|}{ Pulmonary function } \\
\hline $\mathrm{FEV}_{1} / \mathrm{FVC}_{1} \%$ & 38 & $65.3 \pm 7.6$ & 95 & $52.4 \pm 13.0$ & $<0.0001$ \\
\hline $\mathrm{FEV}_{1}, \%$ predicted & 40 & $59.4 \pm 20.3$ & 95 & $41.3 \pm 13.0$ & $<0.0001$ \\
\hline VC, $\%$ predicted & 38 & $71.7 \pm 20.0$ & 95 & $64.4 \pm 16.6$ & 0.034 \\
\hline $\mathrm{RV}, \%$ predicted & 10 & $189.7 \pm 98.1$ & 86 & $204.3 \pm 71.1$ & 0.43 \\
\hline $\mathrm{RV} / \mathrm{TLC}, \%$ & 10 & $168.0 \pm 50.1$ & 86 & $162.0 \pm 31.6$ & 0.90 \\
\hline TLC, $\%$ predicted & 10 & $108.5 \pm 31.6$ & 86 & $114.7 \pm 25.2$ & 0.38 \\
\hline DLCO, \% predicted & 7 & $52.7 \pm 24.3$ & 44 & $33.3 \pm 12.9$ & 0.022 \\
\hline $\mathrm{KCO}, \%$ predicted & 7 & $64.6 \pm 26.3$ & 45 & $48.0 \pm 18.1$ & 0.086 \\
\hline \multicolumn{6}{|l|}{ CPET } \\
\hline Maximum power, $\%$ predicted* & 39 & $63.5 \pm 17.3$ & 95 & $48.4 \pm 17.1$ & $<0.0001$ \\
\hline $\mathrm{VO}_{2}$ peak, $\%$ predicted* & 40 & $72.6 \pm 15.7$ & 95 & $51.6 \pm 15.7$ & $<0.0001$ \\
\hline VO ${ }_{2} @ A T, \%$ predicted* & 36 & $81.9 \pm 17.9$ & 79 & $65.4 \pm 16.6$ & $<0.0001$ \\
\hline $\mathrm{VO}_{2}$ peak/HRpeak, \% predicted* & 39 & $85.6 \pm 16.6$ & 94 & $63.5 \pm 19.5$ & $<0.0001$ \\
\hline VÉpeak, \% predicted* & 39 & $71.6 \pm 20.9$ & 94 & $59.4 \pm 16.2$ & 0.0012 \\
\hline VÉ/MVV, \% & 39 & $68.4 \pm 15.3$ & 93 & $79.7 \pm 18.3$ & 0.0005 \\
\hline BFpeak, breaths/min & 39 & $30 \pm 6$ & 94 & $32 \pm 6$ & 0.18 \\
\hline VÉ/ $\mathrm{VCO}_{2}$ slope & 40 & $33 \pm 9$ & 89 & $37 \pm 9$ & 0.0004 \\
\hline petCO ${ }_{2} @ A T, m m ~ H g$ & 36 & $35.8 \pm 5.8$ & 73 & $32.8 \pm 5.8$ & 0.0042 \\
\hline VÉ/VCO ${ }_{2}$ nadir & 36 & $32.5 \pm 7.4$ & 79 & $38.8 \pm 7.9$ & $<0.0001$ \\
\hline
\end{tabular}

BFpeak, peak breathing frequency; BMI, body mass index; COPD, chronic obstructive pulmonary disease; CPET, cardiopulmonary exercise testing; DLCO, diffusion capacity of the lung for carbon monoxide; $\mathrm{FEV}_{1}$, forced expiratory volume in $1 \mathrm{~s} ; \mathrm{FVC}$, forced vital capacity; KCO, Krogh factor (diffusion capacity of the lung for carbon monoxide per alveolar volume); PetCO $@$ @AT, end-tidal pressure of carbon dioxide at anaerobic threshold; RV, residual volume; SD, standard deviation; TLC, total lung capacity; VC, vital capacity; VÉ/MVV, ratio of ventilation to maximum voluntary ventilation; VÉpeak, peak ventilation; VÉ/ $\mathrm{VCO}_{2}$ nadir, ratio of ventilation to carbon dioxide output; VÉ/VCO 2 slope, slope of the relation between ventilation and carbon dioxide output; $\mathrm{VO}_{2} @ \mathrm{AT}$, oxygen uptake at anaerobic threshold; $\mathrm{VO}_{2}$ peak, peak oxygen uptake; $\mathrm{VO}_{2}$ peak/ $\mathrm{HRpeak}$, ratio of peak oxygen uptake to peak heart rate. ${ }^{*}$ Reference values were calculated as described [29] (see online suppl. Table 1 for details).

Table 6. Two different models for the calculation of predictive variables for survival in multivariate Cox analysis

\begin{tabular}{llcc}
\hline & HR $(95 \% \mathrm{Cl})$ & $\chi^{2}$ & $p$ value \\
\hline Model $1^{*}$ & & & \\
$\quad$ Age, years & $1.031(1.003 ; 1.059)$ & 4.71 & 0.030 \\
$\quad$ Dyspnoea & $1.640(1.173 ; 2.291)$ & 8.39 & 0.0038 \\
$\quad$ VO 2 peak, $\mathrm{mL} / \mathrm{kg} / \mathrm{min}$ & $0.886(0.830 ; 0.946)$ & 12.96 & 0.0003 \\
Model 2 & & & \\
$\quad$ Dyspnoea & $1.519(1.103 ; 2.093)$ & 6.35 & 0.012 \\
$\quad \mathrm{VO}_{2}$ peak, \% predicted & $0.965(0.949 ; 0.981)$ & 18.34 & $<0.0001$ \\
\hline
\end{tabular}

$\mathrm{Cl}$, confidence interval; GOLD, global initiative for chronic obstructive lung disease; $\mathrm{HR}$, hazard ratio; $\mathrm{VO}_{2}$ peak, peak oxygen uptake; $\mathrm{FEV}_{1}$, forced expiratory volume in $1 \mathrm{~s} .{ }^{*}$ Model 1 included age, gender, GOLD, grade, degree of dyspnoea, $\mathrm{VO}_{2}$ peak ( $\mathrm{mL} / \mathrm{kg} / \mathrm{min}$ ), minute ventilation/carbon dioxide output at the anaerobic threshold, and systolic maximum pressure $(\mathrm{mm} \mathrm{Hg}){ }^{\dagger}$ Model 2 included $\mathrm{GOLD}$, grade, degree of dyspnoea, $\mathrm{VO}_{2}$ peak (\% predicted), maximum power (\% predicted), and $\mathrm{FEV}_{1}$ (\% predicted). patients with grade IV COPD. Survival according to GOLD category is shown in online supplementary Figure 1 (see www.karger.com/doi/10.1159/000519750 for all online suppl. material). Survival at 3, 5, and 10 years was $93 \%, 93 \%$, and $62 \%$, respectively, in category A, $83 \%$, $76 \%$, and $73 \%$, respectively, in category $\mathrm{B}, 88 \%, 88 \%$, and $77 \%$, respectively, in category $\mathrm{C}$, and $84 \%, 75 \%$, and $48 \%$, respectively, in category $\mathrm{D}$.

Figure 3 shows survival in relation to the $\mathrm{mBODE}$ index. Survival at 3, 5, and 10 years was $90 \%, 85 \%$, and $78 \%$, respectively, in patients with an $\mathrm{mBODE}$ score of $0-2$,
$92 \%, 86 \%$, and $70 \%$, respectively, in those with an $\mathrm{mBODE}$ score of $3-4,82 \%, 79 \%$, and $51 \%$, respectively, in those with an mBODE score of 5-6, and 77\%, 55\%, and 25\%, respectively, in those with an $\mathrm{mBODE}$ score of 7-10. In an analysis of individual components of the mBODE index (Fig. 4), the degree of patient dyspnoea was found to be an excellent differentiator of survival from the third year onwards. Categories of $\mathrm{VO}_{2}$ peak (\% predicted) also differentiated survival well from the third year onwards (except for the categories $60 \%$ to $<70 \%$ predicted vs. $>40 \%$ to $<60 \%$ predicted). 


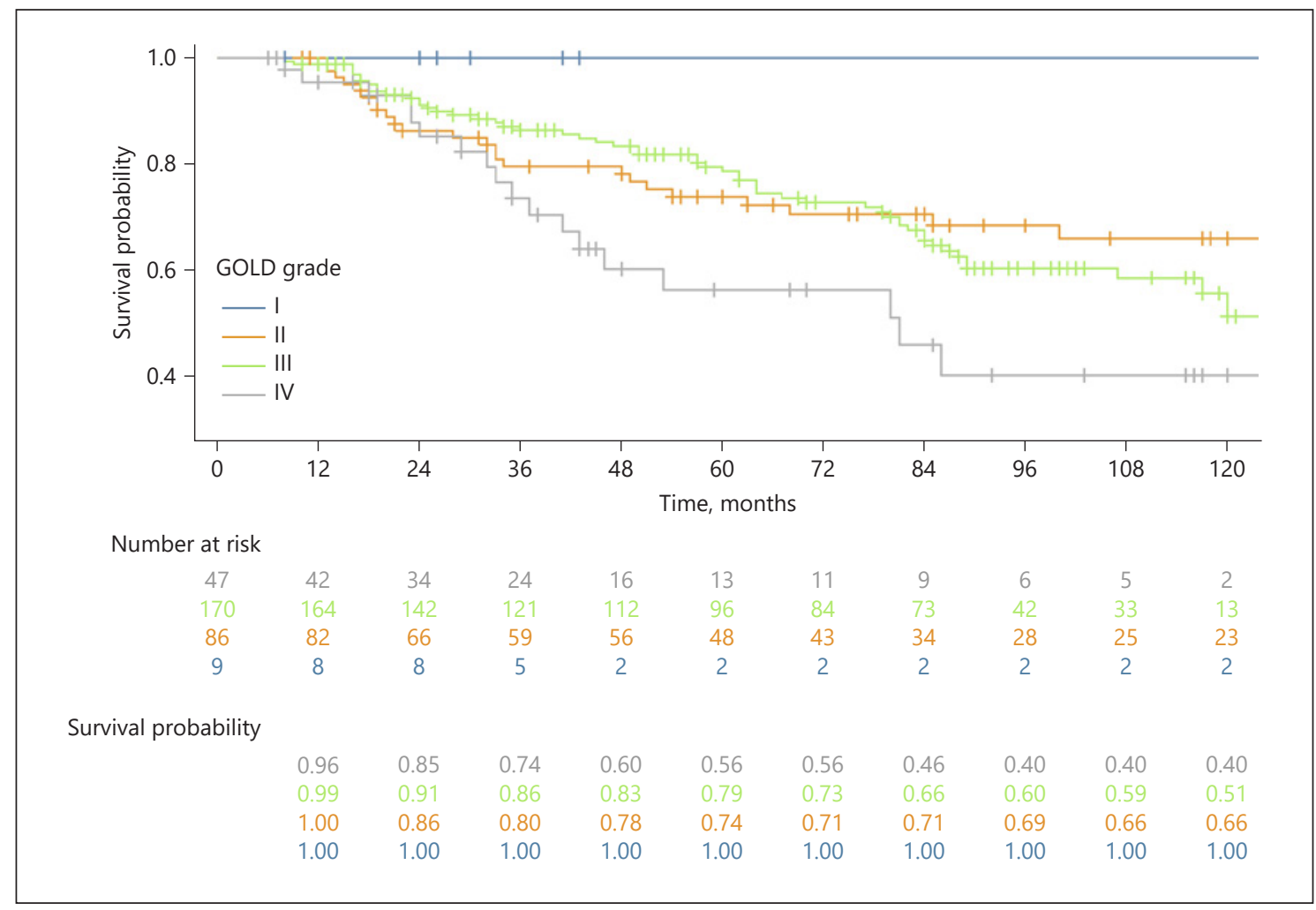

Fig. 2. All-cause mortality according to GOLD grades. GOLD, global initiative for chronic obstructive lung disease.

Fig. 3. Survival according to the $\mathrm{mBODE}$ index $(n=275)$. mBODE, modified BMI, airflow obstruction, dyspnoea, and exercise capacity.

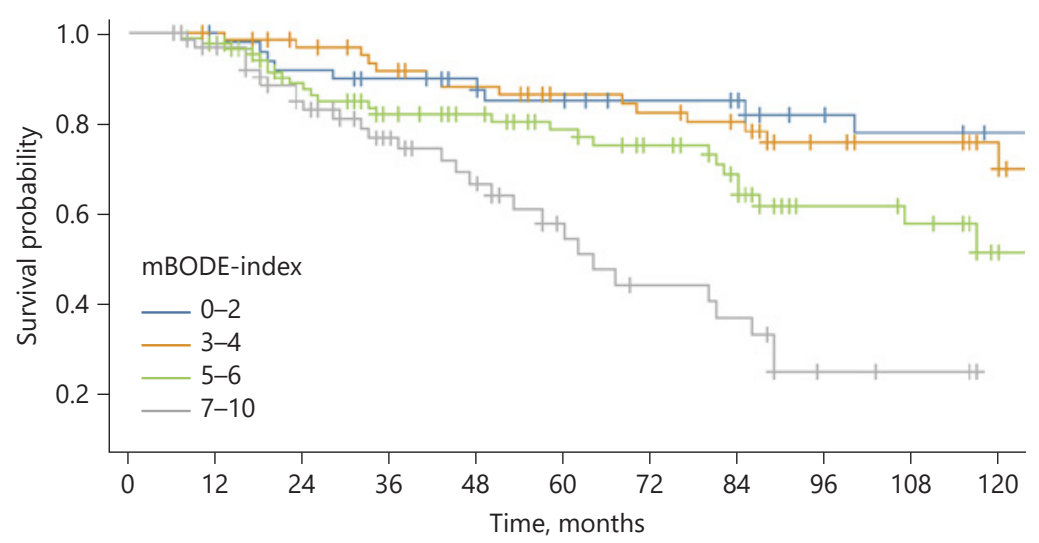

Number at risk

$\begin{array}{ccccccccccc}67 & 60 & 47 & 33 & 26 & 17 & 12 & 10 & 4 & 3 & 0 \\ 90 & 84 & 67 & 57 & 52 & 45 & 38 & 30 & 17 & 15 & 6 \\ 67 & 66 & 61 & 53 & 49 & 44 & 42 & 37 & 26 & 24 & 13 \\ 51 & 49 & 45 & 42 & 38 & 35 & 32 & 28 & 22 & 20 & 18\end{array}$

Survival probability

$\begin{array}{llllllllll}0.97 & 0.83 & 0.77 & 0.67 & 0.55 & 0.44 & 0.37 & 0.25 & 0.25 & 0.25 \\ 0.98 & 0.87 & 0.82 & 0.82 & 0.79 & 0.75 & 0.67 & 0.62 & 0.58 & 0.51 \\ 1.00 & 0.97 & 0.92 & 0.88 & 0.86 & 0.82 & 0.80 & 0.76 & 0.76 & 0.70 \\ 1.00 & 0.92 & 0.90 & 0.87 & 0.85 & 0.85 & 0.85 & 0.82 & 0.78 & 0.78\end{array}$



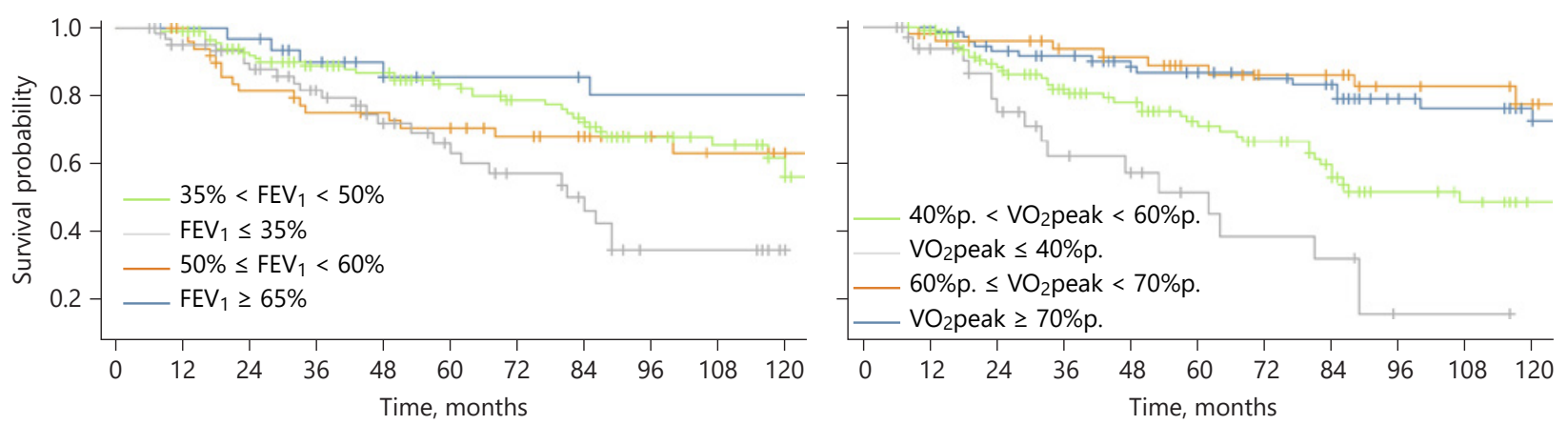

Number at risk

$\begin{array}{ccccccccccc}65 & 58 & 50 & 37 & 28 & 22 & 71 & 13 & 6 & 6 & 1 \\ 123 & 119 & 100 & 87 & 82 & 70 & 62 & 56 & 33 & 29 & 11 \\ 52 & 50 & 39 & 35 & 34 & 32 & 28 & 20 & 15 & 12 & 10 \\ 35 & 32 & 31 & 26 & 21 & 17 & 17 & 16 & 15 & 15 & 15\end{array}$

Survival probability
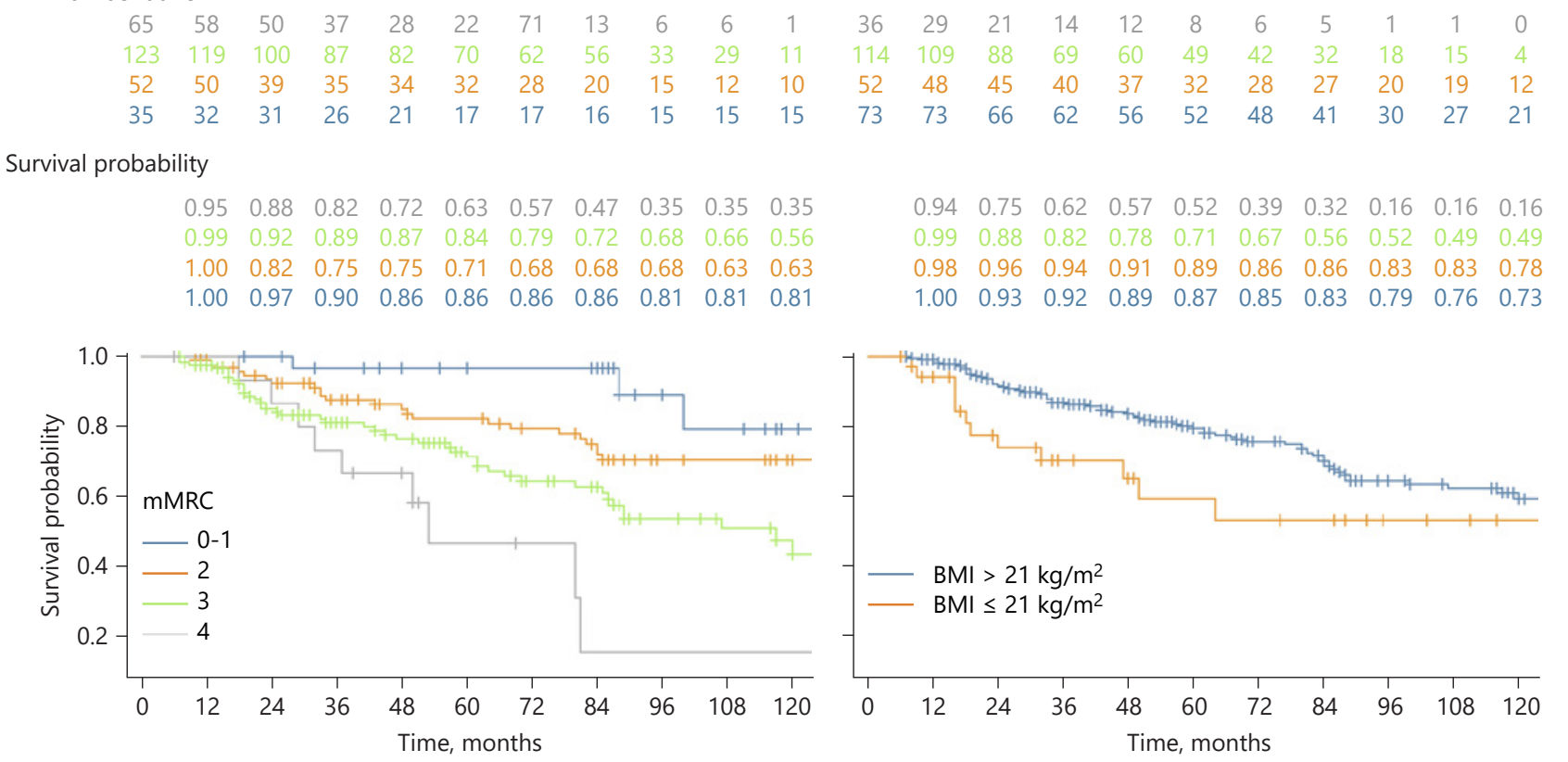

Number at risk

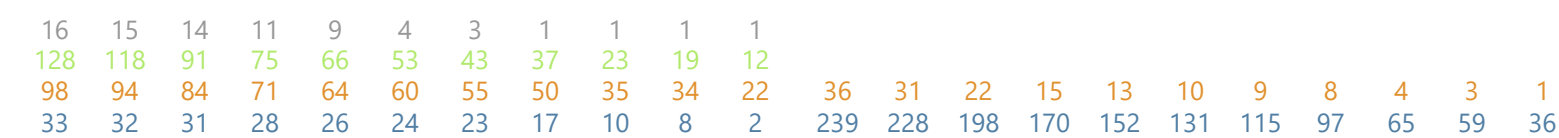

Survival probability

$$
\begin{array}{llllllllll}
1.00 & 0.87 & 0.73 & 0.67 & 0.47 & 0.47 & 0.16 & 0.16 & 0.16 & 0.16 \\
0.98 & 0.85 & 0.81 & 0.77 & 0.71 & 0.64 & 0.63 & 0.54 & 0.51 & 0.44 \\
0.99 & 0.92 & 0.88 & 0.85 & 0.85 & 0.79 & 0.72 & 0.71 & 0.71 & 0.71 \\
1.00 & 1.00 & 0.97 & 0.97 & 0.97 & 0.97 & 0.97 & 0.89 & 0.79 & 0.79
\end{array}
$$

Fig. 4. Survival according to individual components of the mBODE index $(n=275)$. BMI, body mass index; FEV , forced expiratory volume in $1 \mathrm{~s}$; mBODE, modified BMI, airflow obstruction, dyspnoea, and exercise capacity; mMRC, modified medical research council dyspnoea scale; $\mathrm{VO}_{2}$ peak, peak oxygen uptake.

\section{Parameters Relevant to Prognosis}

Multivariable stepwise Cox analysis was performed including significant values in univariate analysis, for example, age, gender, $\mathrm{VO}_{2}$ peak $(\mathrm{mL} / \mathrm{kg} / \mathrm{min})$, COPD grade, degree of dyspnoea, $\mathrm{VÉ} / \mathrm{VCO}_{2}$ nadir, and systolic maxi- mum pressure $(\mathrm{mm} \mathrm{Hg})$. In this analysis, age, degree of dyspnoea, and $\mathrm{VO}_{2}$ peak were independently predictive of survival (Table 6). A second multivariable forward stepwise Cox analysis was performed including $\mathrm{VO}_{2}$ peak (\% predicted), COPD grade, degree of dyspnoea, maximum 

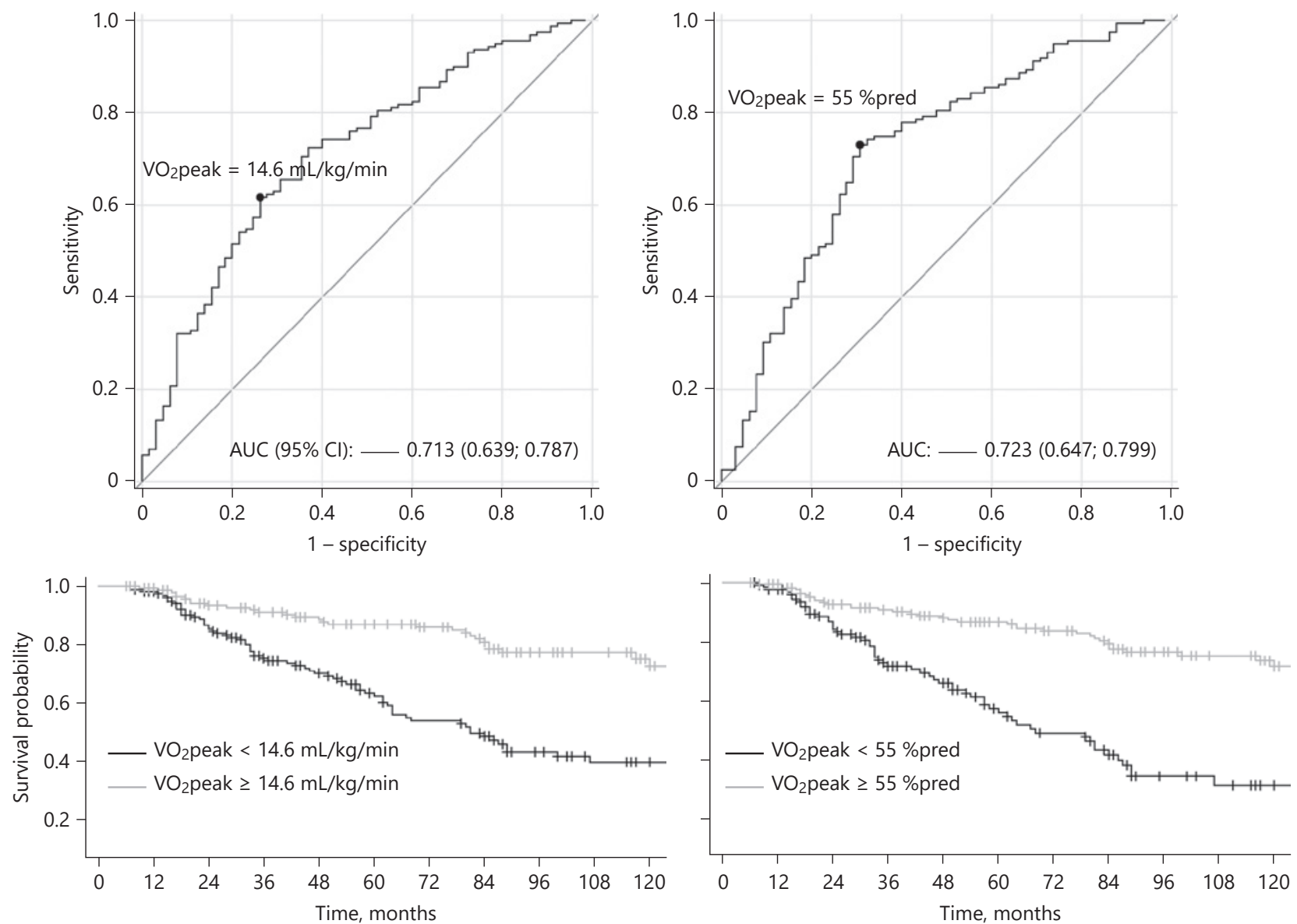

Number at risk

$$
\begin{array}{lllllllllll}
147 & 141 & 126 & 114 & 106 & 98 & 89 & 73 & 50 & 44 & 30 \\
165 & 155 & 124 & 95 & 80 & 61 & 51 & 45 & 28 & 21 & 10
\end{array}
$$

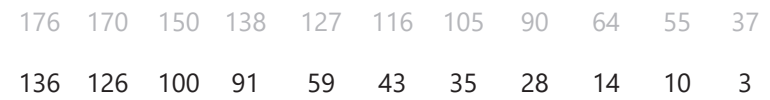

Survival probability

$$
\begin{array}{llllllllll}
0.99 & 0.94 & 0.91 & 0.89 & 0.87 & 0.86 & 0.81 & 0.77 & 0.77 & 0.73 \\
0.98 & 0.85 & 0.75 & 0.70 & 0.62 & 0.54 & 0.49 & 0.43 & 0.40 & 0.40
\end{array}
$$

Fig. 5. $\mathrm{VO}_{2}$ peak for discrimination of survival at 5 years. In ROC analysis (upper panels), $\mathrm{VO}_{2}$ peak $=14.6 \mathrm{~mL} /$ $\mathrm{kg} / \mathrm{min}$ and $\mathrm{VO}_{2}$ peak $=55 \%$ predicted were identified as the optimal thresholds for prediction of survival at 5 years. Kaplan-Meier plots (lower panels) show survival in patient subgroups defined based on these thresholds. AUC, area under the curve (reported with 95\% confidence interval); $\mathrm{VO}_{2}$ peak, peak oxygen uptake; ROC, receiver operating characteristic.

power (\% predicted), $\mathrm{FEV}_{1}$ (\% predicted), and DLCO (\%pred.). In this analysis, degree of dyspnoea and $\mathrm{VO}_{2}$ peak (\% predicted) were independently predictive of survival (Table 6). Backward and forward analysis yielded the same results.

ROC curves for $\mathrm{VO}_{2}$ peak were therefore created to identify the optimal threshold for prediction of 5-year survival (Fig. 5). A $\mathrm{VO}_{2}$ peak of $14.6 \mathrm{~mL} / \mathrm{kg} / \mathrm{min}$ (or $55 \%$ predicted) was an excellent discriminator of survival from the second year onwards. The optimal threshold for prediction of 1- and 3-year survival was $14.3 \mathrm{~mL} / \mathrm{kg} / \mathrm{min}$ (or $44 \%$ predicted at 1 year and $56 \%$ predicted at 3 years) (online suppl. Fig. 2-5). Comparison of ROC curves for $\mathrm{VO}_{2}$ peak (\% predicted) and 3 multidimensional prognos- 

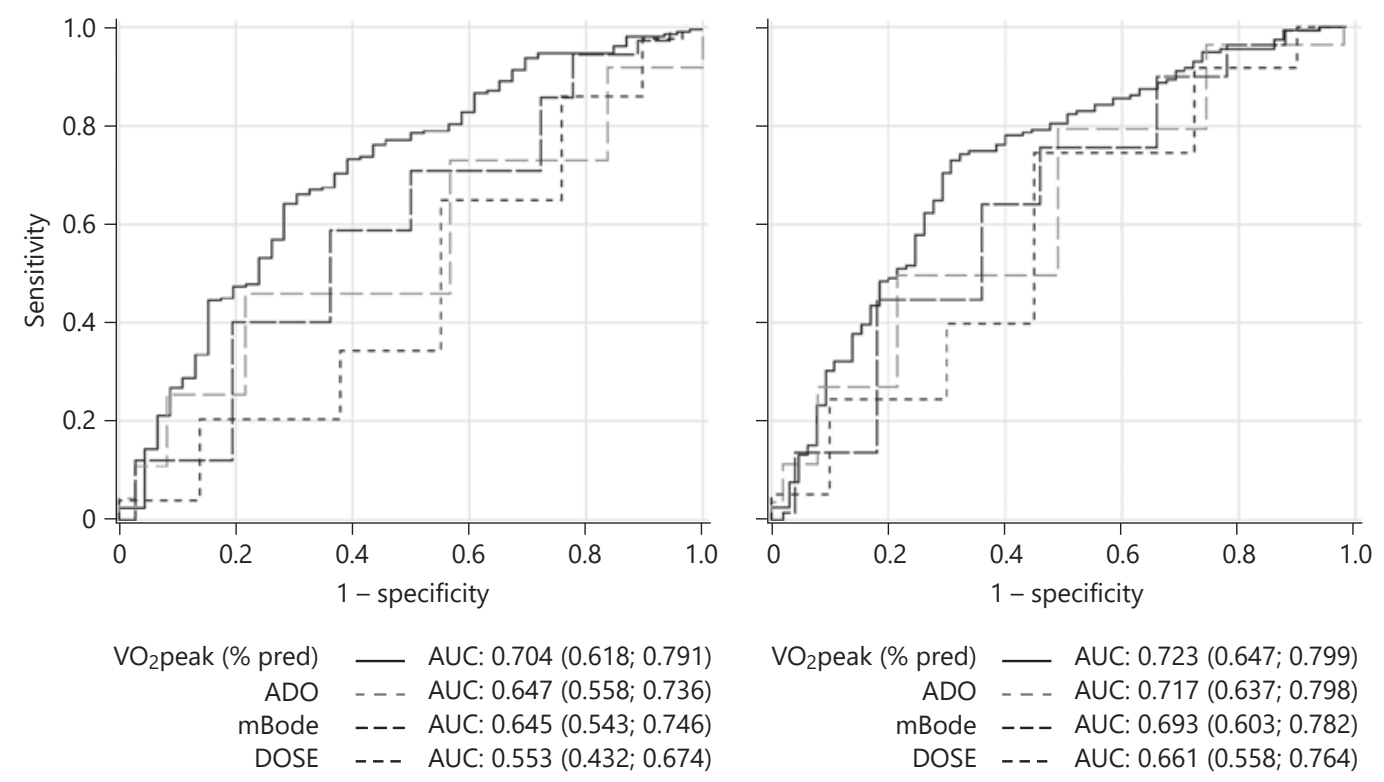

Fig. 6. ROC analysis of $\mathrm{VO}_{2}$ peak and multidimensional prognostic indices for discrimination of survival at 3 (left) and 5 (right) years. ADO, age, dyspnoea, and airflow obstruction; AUC, area under the curve (reported with 95\% confidence interval); DOSE, dyspnoea, airflow obstruction, current smoking status, and exacerbations; mBODE, modified BMI, airflow obstruction, dyspnoea, and exercise capacity; $\mathrm{VO}_{2}$ peak, peak oxygen uptake; ROC, receiver operating characteristic.

tic indices (mBODE, age, dyspnoea, and airflow obstruction, and dyspnoea, airflow obstruction, current smoking status, and exacerbations) demonstrated that $\mathrm{VO}_{2}$ peak had a higher area under the curve (indicating greater discriminative ability) than the multidimensional indices for the prediction of survival at 3 and 5 years (Fig. 6).

Consequently, we analysed whether addition of $\mathrm{VO}_{2}$ peak to the GOLD categories A-D (grouped as A/B and $\mathrm{C} / \mathrm{D}$ because of the low numbers of patients in categories $\mathrm{A}$ and $\mathrm{C}$ ) would improve differentiation of prognosis. The 5-year survival in patients with $\mathrm{VO}_{2}$ peak $<14.6 \mathrm{~mL} /$ $\mathrm{kg} / \mathrm{min}$ versus $\geq 14.6 \mathrm{~mL} / \mathrm{kg} / \mathrm{min}$ was $60 \%$ versus $86 \%$ in Group A/B and $64 \%$ versus $90 \%$ in Group C/D. In a comparable analysis of patients with $\mathrm{VO}_{2}$ peak $<55 \%$ predicted versus $\geq 55 \%$ predicted, 5 -year survival was $0 \%$ versus $85 \%$ in Group A/B and 61\% versus 90\% in Group C/D (Fig. 7).

\section{Discussion}

Our study of 312 patients with COPD and a mean follow-up of $>5$ years demonstrates clearly that $\mathrm{VO}_{2}$ peak, a surrogate marker for cardiopulmonary exercise capacity, has a highly significant influence on survival. Our results suggest that the assessment of prognosis in patients with COPD could be further improved by the addition of $\mathrm{VO}_{2}$ peak to established prognostic indicators.

Survival probability in our study population $(75 \%$ and $57 \%$ after 5 and 10 years, respectively) reflects the impaired prognosis of patients with COPD and was somewhat lower than reported previously in smaller studies by Hiraga et al. [19] (86\% and 68\% after 5 and 10 years, respectively; $n=120$ ), Tojo et al. [22] (93\% and $76 \%$ after 5 and 10 years, respectively; $n=69)$, and Oga et al. [20] ( $80 \%$ at 5 years; $n=150)$. The mean age, gender distribution, $\mathrm{FEV}_{1}$, and $\mathrm{VO}_{2}$ peak in our study population were within the range reported in the 3 previous studies $[19,20,22]$; a possible explanation for the difference in survival could be geographical variation (the previous studies were conducted in Japan). Survival at 3 and 5 years stratified by mBODE score was broadly comparable with data from a previous study of $444 \mathrm{pa}-$ tients with COPD in the USA and Spain (which reported 3 - and 5-year survival of $93 \%$ and $89 \%$, respectively, in those with an mBODE score of $0-2$ points, $89 \%$ and $81 \%$, respectively, in those with an $\mathrm{mBODE}$ score of 3-4 


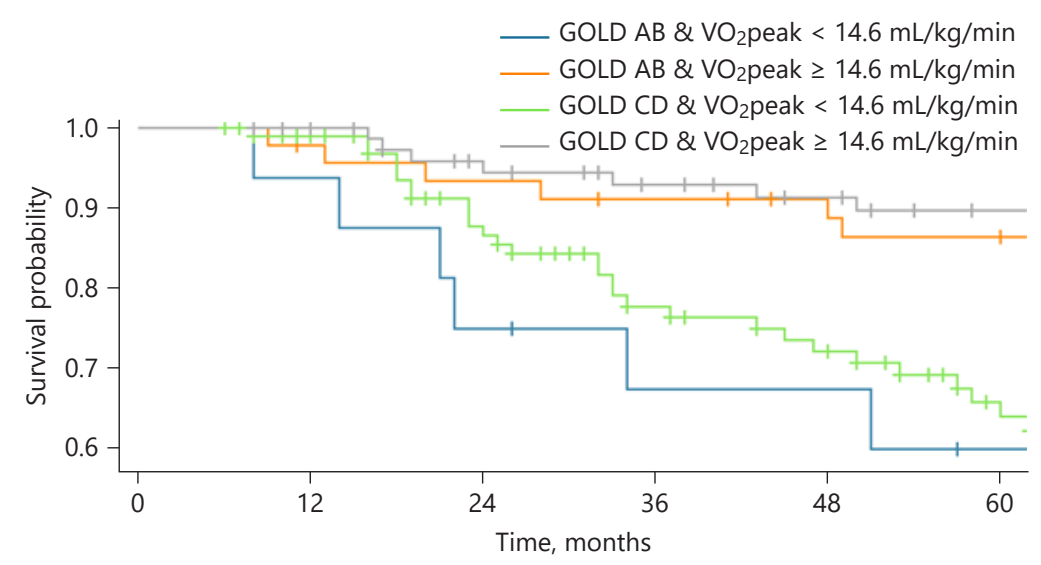

Number at risk

$\begin{array}{cccccc}77 & 75 & 67 & 60 & 56 & 51 \\ 101 & 94 & 76 & 59 & 51 & 37 \\ 48 & 44 & 42 & 40 & 38 & 36 \\ 16 & 15 & 12 & 9 & 9 & 7\end{array}$

Survival probability

$\begin{array}{lllll}1.00 & 0.94 & 0.93 & 0.91 & 0.90 \\ 0.99 & 0.87 & 0.78 & 0.72 & 0.64 \\ 0.98 & 0.93 & 0.91 & 0.89 & 0.86 \\ 0.94 & 0.75 & 0.68 & 0.68 & 0.60\end{array}$

Fig. 7. Kaplan-Meier analysis of survival in patients stratified by GOLD category and $\mathrm{VO}_{2}$ peak. GOLD, global initiative for chronic obstructive lung disease; $\mathrm{VO}_{2}$ peak, peak oxygen uptake.

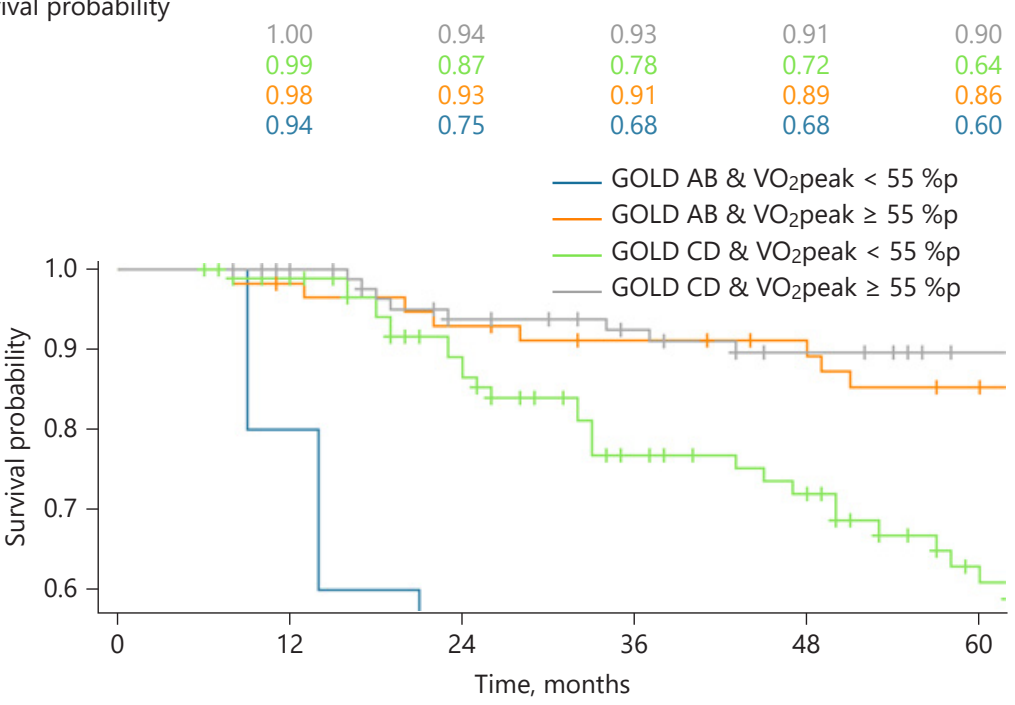

Number at risk

$\begin{array}{cccccc}86 & 83 & 73 & 67 & 62 & 57 \\ 92 & 86 & 70 & 52 & 45 & 31 \\ 58 & 55 & 52 & 49 & 47 & 43 \\ 6 & 4 & 2 & 0 & & \end{array}$

Survival probability

$\begin{array}{lllll}1.00 & 0.94 & 0.92 & 0.90 & 0.90 \\ 0.99 & 0.86 & 0.77 & 0.72 & 0.61 \\ 0.98 & 0.93 & 0.91 & 0.89 & 0.85 \\ 0.80 & 0.40 & 0.00 & & \end{array}$

points, $85 \%$ and $66 \%$, respectively, in those with an mBODE score of 5-6 points, and $70 \%$ and $54 \%$, respectively, in those with an mBODE score of 7-10 points) [18].

Cardiopulmonary Exercise Testing in COPD Patients
Recommendations for the management of COPD suggest that multidimensional indices such as BODE allow a better assessment of prognosis than individual parameters $[2,25]$. However, $\mathrm{VO}_{2}$ peak demonstrated superiority 
Fig. 8. Suggested inclusion of $\mathrm{VO}_{2}$ peak in the GOLD ABCD refined assessment tool. COPD, chronic obstructive pulmonary disease assessment test; GOLD, global initiative for chronic obstructive lung disease; mMRC, modified medical research council dyspnoea scale; $\mathrm{VO}_{2}$ peak, peak oxygen uptake.

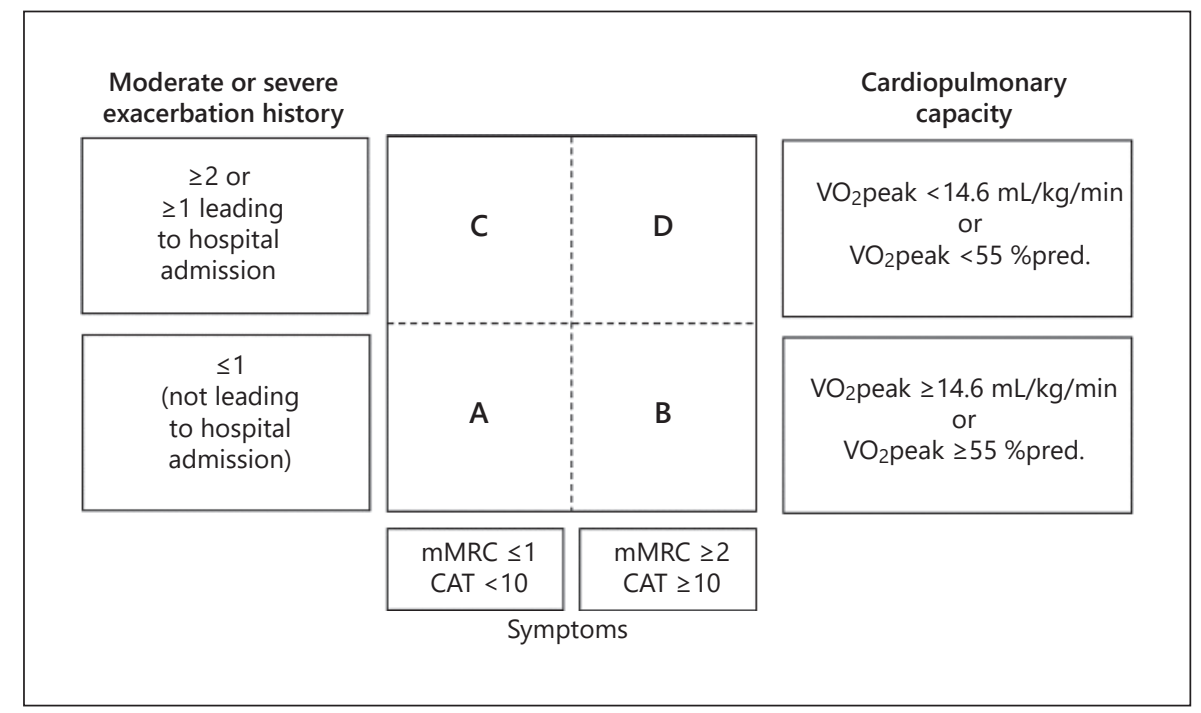

to specific multidimensional indices for the prediction of mortality in a previous analysis of 150 male patients with COPD [32], and in our study of 312 patients ( $74 \%$ male) with COPD. According to the GOLD 2020 recommendations, assessment of the severity of COPD is based on spirometric classification (GOLD grades I-IV) combined with categorization based on symptoms and history of moderate or severe exacerbations (GOLD categories $\mathrm{A}-\mathrm{D})$ [2]. We would like to suggest including $\mathrm{VO}_{2}$ peak in the categorization tool to improve estimation of prognosis if prospective studies are available (Fig. 8).

In our prognosis models, dyspnoea and age were also significantly associated with survival. It has long been known that the degree of dyspnoea has significantly more prognostic relevance than grading according to lung function values in patients with COPD [33-36]. Dyspnoea is a substantial correlate of hyperinflation in patients with COPD, which often results in dynamic hyperinflation and disturbance of ventilatory efficiency [2]. A significant association of age with mortality in patients with COPD has also been observed in previous studies $[19,20,22]$. Several studies have shown that ventilatory insufficiency is characteristic of COPD patients [37], and in some of these its prognostic value could be demonstrated [24]. The present study could demonstrate the significant influence of $\mathrm{VE} / \mathrm{VCO}_{2}$ nadir on prognosis by univariate calculation albeit not by multivariable analysis.

Even though the method seems time-consuming (approximately $45 \mathrm{~min}$ per patient), it may deliver many relevant information on cardiopulmonary performance and may lead to further examinations (echocardiography, left/right heart catheter). Further, it is the only examination method that may indicate the existence of dynamic hyperinflation.

Limitations include the retrospective nature of the study and a relevant bias in the selection of patients, as only patients who had undergone CPET were included for evaluation. The set of history, clinical, and functional parameters could not completely be documented in all patients. Echocardiographic and 6-min walking distance data were not available for the present analyses. Therefore, the coincident existence of pulmonary hypertension cannot completely be ruled out. However, patients with obvious clinical aspects of right heart overload were excluded from analysis.

All patients followed a single CPET protocol; however, $\mathrm{VO}_{2}$ peak was not significantly altered by the use of different CPET protocols in previous studies of patients with COPD [38, 39]. Established parameters of ventilatory mechanics (e.g., dynamic hyperinflation, inspiratory capacity) were not available for analysis. These and other parameters (e.g., ventilatory inefficiency) taken together allow even better to depict the complex pathophysiology of COPD [34, 40, 41]. Especially the parameter of VE/ $\mathrm{VCO}_{2}$ slope is influenced by the degree of COPD and is therefore being underestimated in advanced stages of the disease [37]. The probably, in this respect, even better parameter of $\mathrm{VÉ} / \mathrm{VCO}_{2}$ intercept was not available for analysis in our study. In $265 / 312$ of patients, we were able to determine the anaerobic threshold [42]. It is a well-known fact that some COPD patients do not reach the anaerobic threshold because of mechanical (pulmonary) limitations during exercise. 
In conclusion, we confirm in a large study population with a substantial duration of follow-up that $\mathrm{VO}_{2}$ peak is a highly significant predictor of survival in patients with COPD. Our data support the incorporation of this surrogate marker of impaired cardiopulmonary capacity into the clinical assessment of patients with COPD alongside established markers $\left(\mathrm{FEV}_{1}\right.$, exacerbation frequency, and symptoms). Prospective studies are needed to confirm our findings with regard to the prognostic relevance of exercise data.

\section{Acknowledgments}

We thank the staff of participating practices and institutions, especially Ms Mennel, Dagmar Fimmel, Kathrin Gutsche, Jeannette Pieper, Mandy Lüdemann, and Claudia Koselowski. Dr Claire Mulligan, PhD (Beacon Medical Communications Ltd, Brighton, UK) provided editorial support, funded by the University of Greifswald.

\section{Statement of Ethics}

The study was approved by the ethical committee of the University of Greifswald (Reg.-Nr. BB 051/2018, April 01, 2018). All patients were informed of the study either by phone or during follow-up visits, and those participating provided written informed consent for the collection of data.

\section{Conflict of Interest Statement}

R.E. received honoraria for scientific lectures from Actelion, GSK, United Therapeutics, AstraZeneca, Novartis, Berlin-Chemie, Boehringer Ingelheim, and OMT, and research funding from Boehringer Ingelheim and Actelion, Germany. M.H. received per- sonal fees for lectures and consultations and travel, accommodation, and/or meeting expenses from Actelion, AOPOrphan/OMT, AstraZeneca, Bayer, Berlin-Chemie, Boehringer, GILEAD, GSK, MSD, Novartis, and Pfizer. G.H. received honoraria for scientific lectures from GSK, AstraZeneca, Novartis, Berlin-Chemie, and Boehringer Ingelheim. S.G. received honoraria for scientific lectures from AstraZeneca, Novartis, Berlin-Chemie, Boehringer Ingelheim, and Roche. A.O., A.Mü, J.W., B.T., A.Ho, M.W., A.He, A.Ma, B.S: No potential conflict of interest to report.

\section{Funding Sources}

This study had no funding. All authors had full access to all the data in the study and accept responsibility for the decision to submit for publication.

\section{Author Contributions}

R.E., S.G., and B.S. were involved in the study conception. R.E., S.G., and A.O. were involved in the study design. A.Mü, M.H., J.W., B.T., G.H., A.Ho, M.W, A.He, and A.Ma included patients in this study. A.O. was the study statistician. R.E., S.G., G.H., B.S., A.Mü, and A.O were involved in the analysis. B.S., R.E., G.H., and A.O. were involved in interpreting the data. R.E. and B.S. wrote the first draft. All authors read and revised the draft of the work. All authors read and approved the final manuscript. Claire Mulligan $\mathrm{PhD}$ (Beacon Medical Communications Ltd, Brighton, UK) edited the final draft of the manuscript prepared by the authors.

\section{Data Availability Statement}

With publication, we declare to make data (deidentified participant data, data dictionary, and statistical analysis plan) if requested available. This should be done through our statisticians Dr. Anne Obst (anne.obst@med.uni-greifswald.de) and based upon a data access agreement.

\section{References}

1 Soriano JB, Abajobir AA, Abate KH, Abera SF, Agrawal A, Ahmed MB, et al. Global, regional, and national deaths, prevalence, disability-adjusted life years, and years lived with disability for chronic obstructive pulmonary disease and asthma, 1990-2015: a systematic analysis for the Global Burden of Disease Study 2015. Lancet Respir Med. 2017;5(9): 691-706.

2 Global Initiative for Chronic Obstructive Lung Disease. Global strategy for the diagnosis, management, and prevention of chronic obstructive pulmonary disease (2020 report). Global Initiative for Chronic Obstructive Lung Disease; 2020. Available from: https:// goldcopd.org/gold-reports/.
3 Mannino DM, Thorn D, Swensen A, Holguin F. Prevalence and outcomes of diabetes, hypertension and cardiovascular disease in COPD. Eur Respir J. 2008;32(4):962-9.

4 Sode BF, Dahl M, Nordestgaard BG. Myocardial infarction and other co-morbidities in patients with chronic obstructive pulmonary disease: a Danish nationwide study of 7.4 million individuals. Eur Heart J. 2011;32(19): 2365-75.

5 Cavailles A, Brinchault-Rabin G, Dixmier A, Goupil F, Gut-Gobert C, Marchand-Adam S, et al. Comorbidities of COPD. Eur Respir Rev. 2013;22(130):454-75.
6 Young KA, Regan EA, Han MK, Lutz SM, Ragland M, Castaldi PJ, et al. Subtypes of COPD have unique distributions and differential risk of mortality. Chronic Obstr Pulm Dis. 2019;6(5):400-13.

7 Watz H, Pitta F, Rochester CL, Garcia-Aymerich J, ZuWallack R, Troosters T, et al. An official European respiratory society statement on physical activity in COPD. Eur Respir J. 2014;44(6):1521-37.

8 Garcia-Aymerich J, Lange P, Benet M, Schnohr $\mathrm{P}$, Antó JM. Regular physical activity modifies smoking-related lung function decline and reduces risk of chronic obstructive pulmonary disease: a population-based cohort study. Am J Respir Crit Care Med. 2007;175(5):458-63. 
9 Vaes AW, Garcia-Aymerich J, Marott JL, Benet M, Groenen MT, Schnohr P, et al. Changes in physical activity and all-cause mortality in COPD. Eur Respir J. 2014;44(5): 1199-209.

10 Westhoff M, Litterst P, Ewert R. Cardiopulmonary exercise testing in combined pulmonary fibrosis and emphysema. Respiration. 2021;100(5):395-403.

11 Ferrazza AM, Martolini D, Valli G, Palange P. Cardiopulmonary exercise testing in the functional and prognostic evaluation of patients with pulmonary diseases. Respiration. 2009;77(1):3-17.

12 Verhage TL, Vercoulen JH, van Helvoort HA, Peters JB, Molema J, Dekhuijzen PN, et al. Maximal exercise capacity in chronic obstructive pulmonary disease: a limited indicator of the health status. Respiration. 2010;80(6): 453-62.

13 Wallaert B, Guetta A, Wemeau-Stervinou L, Tercé G, Valette M, Nevière R, et al. [Prognostic value of clinical exercise testing in idiopathic pulmonary fibrosis]. Rev Mal Respir. 2011;28(3):290-6.

14 Armstrong HF, Thirapatarapong W, Dussault NE, Bartels MN. Distinguishing pulmonary hypertension in interstitial lung disease by ventilation and perfusion defects measured by cardiopulmonary exercise testing. Respiration. 2013;86(5):407-13.

15 van Gestel AJ, Baty F, Rausch-Osthof AK, Brutsche MH. Cardiopulmonary and gas-exchange responses during the six-minute walk test in patients with chronic obstructive pulmonary disease. Respiration. 2014;88(4):30714.

16 Hebestreit H, Arets HG, Aurora P, Boas S, Cerny F, Hulzebos EH, et al. Statement on exercise testing in cystic fibrosis. Respiration. 2015;90(4):332-51.

17 Marin JM, Alfageme I, Almagro P, Casanova C, Esteban C, Soler-Cataluña JJ, et al. Multicomponent indices to predict survival in COPD: the COCOMICS study. Eur Respir J. 2013;42(2):323-32.

18 Cote CG, Pinto-Plata VM, Marin JM, Nekach H, Dordelly LJ, Celli BR. The modified BODE index: validation with mortality in COPD. Eur Respir J. 2008;32(5):1269-74.

19 Hiraga T, Maekura R, Okuda Y, Okamoto T, Hirotani A, Kitada S, et al. Prognostic predictors for survival in patients with COPD using cardiopulmonary exercise testing. Clin Physiol Funct Imaging. 2003;23(6):324-31.

20 Oga T, Nishimura K, Tsukino M, Sato S, Hajiro T. Analysis of the factors related to mortality in chronic obstructive pulmonary disease: role of exercise capacity and health sta- tus. Am J Respir Crit Care Med. 2003;167(4): 544-9.

21 Neder JA, Alharbi A, Berton DC, Alencar MC, Arbex FF, Hirai DM, et al. Exercise ventilatory inefficiency adds to lung function in predicting mortality in COPD. COPD. 2016; 13(4):416-24.

22 Tojo N, Ichioka M, Chida M, Miyazato I, Yoshizawa Y, Miyasaka N. Pulmonary exercise testing predicts prognosis in patients with chronic obstructive pulmonary disease. Intern Med. 2005;44(1):20-5.

23 Çiftçi F, Şen E, Akkoca Yıldız Ö, Saryal S. Does exercise capacity, dyspnea level, or quality of life actually predict mortality in patients with COPD? 8-year follow-up. Tuberk Toraks. 2019;67(2):83-91.

24 Alencar MC, Arbex FF, Souza A, Mazzuco A, Sperandio PA, Rocha A, et al. Does exercise ventilatory inefficiency predict poor outcome in heart failure patients with COPD? J Cardiopulm Rehabil Prev. 2016;36(6):454-9.

25 Celli BR, Wedzicha JA. Update on clinical aspects of chronic obstructive pulmonary disease. N Engl J Med. 2019;381(13):1257-66.

26 Cotes JE, Chinn DJ, Quanjer PH, Roca J, Yernault JC. Standardization of the measurement of transfer factor (diffusing capacity). Eur Respir J. 1993;6(Suppl 16):41-52.

27 Crièe C-P, Berdel D, Heise D, Kardos P, Köhler D, Leupold W, et al. Empfehlungen der deutschen atemwegsliga zur spirometrie. Pneumologie. 2006;60:576-84.

28 Crièe CP, Berdel D, Heise D, Jörres RA, Kardos P, Köhler D, et al. Empfehlungen der Deutschen Atemwegsliga und der Deutschen Gesellschaft für Pneumologie und Beatmungsmedizin. Empfehlungen zur Ganzkörperplethysmographie (Bodyplethysmographie). Munich, Germany and Orlando, USA: Dustri-Verlag Dr. Karl Feistle; 2009. Available from: https://www.atemwegsliga.de/ empfehlungen-positionspapiere.html.

29 Glaser S, Ittermann T, Schaper C, Obst A, Dorr M, Spielhagen T, et al. [The study of health in pomerania (SHIP) reference values for cardiopulmonary exercise testing]. Pneumologie. 2013;67(1):58-63.

30 Puhan MA, Garcia-Aymerich J, Frey M, ter Riet G, Antó JM, Agustí AG, et al. Expansion of the prognostic assessment of patients with chronic obstructive pulmonary disease: the updated BODE index and the ADO index. Lancet. 2009;374(9691):704-11.

31 Jones RC, Donaldson GC, Chavannes NH, Kida K, Dickson-Spillmann M, Harding S, et al. Derivation and validation of a composite index of severity in chronic obstructive pulmonary disease: the DOSE Index. Am J
Respir Crit Care Med. 2009;180(12):118995.

32 Oga $\mathrm{T}$, Tsukino $\mathrm{M}$, Hajiro $\mathrm{T}$, Ikeda $\mathrm{A}$, Nishimura K. Predictive properties of different multidimensional staging systems in patients with chronic obstructive pulmonary disease. Int J Chron Obstruct Pulmon Dis. 2011;6:521-6.

33 Nishimura K, Izumi T, Tsukino $M$, Oga $T$. Dyspnea is a better predictor of 5-year survival than airway obstruction in patients with COPD. Chest. 2002;121(5):1434-40.

34 Kagawa H, Miki K, Kitada S, Miki M, Yoshimura K, Oshitani $Y$, et al. Dyspnea and the varying pathophysiologic manifestations of chronic obstructive pulmonary disease evaluated by cardiopulmonary exercise testing with arterial blood analysis. Front Physiol. 2018;9:1293.

35 Gil HI, Zo S, Jones PW, Kim BG, Kang N, Choi $\mathrm{Y}$, et al. Clinical characteristics of COPD patients according to COPD assessment test (CAT) score level: cross-sectional study. Int J Chron Obstruct Pulmon Dis. 2021;16:1509-17.

36 O'Donnell DE, Elbehairy AF, Faisal A, Webb KA, Neder JA, Mahler DA. Exertional dyspnoea in COPD: the clinical utility of cardiopulmonary exercise testing. Eur Respir Rev. 2016;25(141):333-47.

37 Neder JA, Arbex FF, Alencar MC, O'Donnell $\mathrm{CD}$, Cory J, Webb KA, et al. Exercise ventilatory inefficiency in mild to end-stage COPD. Eur Respir J. 2015;45(2):377-87.

38 Benzo RP, Paramesh S, Patel SA, Slivka WA, Sciurba FC. Optimal protocol selection for cardiopulmonary exercise testing in severe COPD. Chest. 2007;132(5):1500-5.

39 Ewert R, Gläser S, Winkler J, Schrader H, Trümper BG, Haase PU, et al. [Cardiopulmonary exercise testing (CPET) in severe COPD: a multicentre comparison of two test protocols]. Pneumologie. 2012;66(7):402-7.

40 Neder JA, Berton DC, Marillier M, Bernard AC, O'Donnell DE, Canadian Respiratory Research N. Inspiratory constraints and ventilatory inefficiency are superior to breathing reserve in the assessment of exertional dyspnea in COPD. COPD. 2019;16(2):174-81.

41 Maekura R, Hiraga T, Miki K, Kitada S, Yoshimura K, Miki M, et al. Differences in physiological response to exercise in patients with different COPD severity. Respir Care. 2014; 59(2):252-62.

42 Binder RK, Wonisch M, Corra U, Cohen-Solal A, Vanhees L, Saner H, et al. Methodological approach to the first and second lactate threshold in incremental cardiopulmonary exercise testing. Eur J Cardiovasc Prev Rehabil. 2008;15(6):726-34. 